\title{
POLAR COORDINATES AND UT1 - UTC FROM PZT OBSERVATIONS
}

\author{
D. Djurovic \\ Department of Astronomy \\ Belgrade, Yugoslavia
}

\section{INTRODUCTION}

Use of new techniques (Doppler, laser, VLBI) has yielded such results that many astronomers believe that in the near future these techniques will replace classical instruments for observations of the Earth's rotation. None of the modern techniques has furnished observational series which demonstrate that systematic errors in the polar coordinates and UT1 are stable over sufficiently long intervals. Investigations of known phenomena such as sudden changes of the secular term in UT1 - UTC (Munk and MacDonald, 1960), changes of the amplitude and phase of the seasonal irregularities (Fliegel and Hawkins, 1967; Pavlov and Staritzin, 1962), secular motion of the mean pole (Mihailov, 1971; Markowitz, 1960), continental drift (Stoyko, 1938; Djurovic, 1976), quasi-diurnal nutation of Molodenskij (Popov, 1963; Toomre, 1974; Rochester et al., 1974), etc., are complicated by the existence of systematic error variations (accuracy), and to a smaller degree, on the accidental errors (precision). The BIH and IPMS make use of individual series of astronomical latitude, $\phi_{i}$, and (UTO - UTC) $i$ from up to 82 classical instruments (Guinot, 1976). If the systematic errors in these series are independent, the polar coordinates and UT1 - UTC determined from a combination of observational series would result in an improvement of accuracy by at least one order of magnitude through the mutual compensation of the variation in systematic errors. At present, however, it is uncertain whether mutual independence of modern observations (a basic condition for mutual compensation of errors) will be better than that of the classical instruments.

Accordingly, the continuation of parallel observations by new and classical techniques remains indispensable as long as there is no definite proof that $x, y$, and UT1 - UTC obtained by the new techniques are more homogeneous than the results achieved by classical methods. 


\section{POLAR COORDINATES AS OBTAINED BY THREE TYPES OF INSTRUMENTS}

Observational data used for the computation of $\mathrm{x}$ and $\mathrm{y}$ are the instantaneous latitudes, $\phi_{i}$, observed in the period 1967-1971 and communicated to the BIH by 42 observatories. Table A of a previous paper (Djurovic, 1975a) lists the observatories considered. The polar coordinates are computed according to methods described previously (Djurovic, 1975a; Djurovic, 1975b). The only difference is that the computations are carried out separately for three types of instruments (astrolabe - A, photographic zenith tube - PZT, and zenith telescope - 1z).

Yearly means of the differences of our results and the corresponding results of the IPMS, smoothed using Vondrak's method, $\Delta x=x-x$ (IPMS) and $\Delta y=y-y(I P M S)$ are given in Table 1. Variations of the differences, $\triangle x(P Z T)$ and $\triangle y(P Z T)$, appear somewhat larger than those of the other two types of instruments. This is explained by the fact that the number of PZTs (8) are lower than that of the astrolabes and zenith telescopes. With 15 PZTs in operation in 1975 it is surely possible to attain a

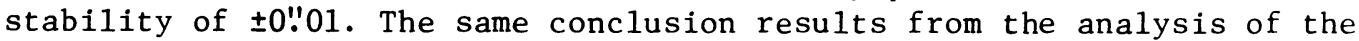
stability of the reference system by the Wilcoxon method (Djurovic, 1978).

The second important condition satisfied by $x(P Z T)$ and $y(P Z T)$ is that their deviations from $x$ (IPMS) and $y$ (IPMS) do not contain periodic terms with periods close to that of the Chandler or annual period as, for example, $\Delta x=x(B I H)-x$ (IPMS). Standard deviations $\varepsilon_{x}$ and $\varepsilon_{y}$ for the PZT solutions are somewhat less than those for the BIH coordinates, but it should be kept in mind that the former are associated with 20-day intervals while the latter are related to 5-day intervals. The values for the period 1967-1971 are:

$$
\begin{array}{ll}
\varepsilon_{\mathrm{x}}(\mathrm{PZT})= \pm 0 ! 015, & \varepsilon_{\mathrm{x}}(\mathrm{BIH})= \pm 0 !^{\prime} 017, \\
\varepsilon_{\mathrm{y}}(\mathrm{PZT})= \pm 0.014, & \varepsilon_{\mathrm{y}}(\mathrm{BIH})= \pm 0.018 .
\end{array}
$$

\section{THE ACCURACY OF THE MEAN SYSTEM (UT1 - UTC) PZT}

Observational material used in the present analysis is from the observations of UTO - UTC at 56 observatories reported to the BIH for the period 1967-1974. All details of the data processing and of the mean system (UT1 - UTC) $u$ are set forth in a previous paper (Djurovic, 1975b). For the observatories H, G, MS, MCP, N, TO, and W, whose series are uninterrupted in the interval 1967-1974 we have computed a system of differences,

$$
d_{i j}=(U T 1-U T C)_{i j}-(U T 1-U T C)_{u},
$$

where (UT1 - UTC) $i j$ is the result of observatory, $i$, on the night of observation, j. From 1971-1974 three more observatories, CL, OS, and RCP, were included. Mean values of $d_{i j}$, denoted by $D_{i}$, have been calculated for 30-day intervals for each observatory, $i$. Two mean systems of $D_{i}$ were calculated: for the group of the first 7 observatories ( $\mathrm{Z}$ ), and for the group of all 10 observatories ( $\left.Z^{\prime}\right)$. The results obtained reveal that $Z$ and $Z^{\prime}$ are varying within the limits of a few milliseconds. 
They do not contain periodic components with periods close to the annual or semiannual terms which would impede the study of the seasonal irregularities in the Earth's rotation.

Table 1. Differences in the polar coordinates for different types of instruments.

\begin{tabular}{|c|c|c|c|c|c|c|}
\hline & $\Delta x(A)$ & $\Delta \mathrm{x}(\mathrm{PZT})$ & $\Delta x(L Z)$ & $\Delta y(A)$ & $\triangle \mathrm{y}(\mathrm{PZT})$ & $\Delta y(L Z)$ \\
\hline 1967 & $-0 . " 049$ & $-0 ! " 032$ & $-0 ! " 030$ & 0.'055 & 0":025 & $-0 . " 009$ \\
\hline 1968 & -0.044 & -0.075 & -0.016 & 0.056 & 0.034 & -0.023 \\
\hline 1969 & -0.046 & -0.086 & -0.022 & 0.077 & 0.037 & -0.014 \\
\hline 1970 & -0.047 & -0.058 & -0.010 & 0.088 & 0.062 & -0.033 \\
\hline 1971 & -0.033 & -0.034 & -0.014 & 0.071 & 0.089 & -0.026 \\
\hline mean & -0.044 & -0.057 & -0.018 & 0.069 & 0.049 & -0.021 \\
\hline
\end{tabular}

Accidental errors were estimated by the expressions:

$$
\begin{aligned}
\sigma^{2} & =\frac{1}{2(N-1)} \sum_{k=2}^{N}\left(z_{k}-Z_{k-1}\right)^{2}, \text { and } \\
\varepsilon_{i}^{2} & =\sum_{j} p_{i j}\left(d_{i j}-D_{i}\right)
\end{aligned}
$$

where $k$ refers to the 30 -day interval, and $p_{i j}$ is the weignt of $d_{i j}$. The results obtained for $\sigma$ and $\varepsilon$ (mean of $\varepsilon_{i}$ ) are $\sigma= \pm 0$ \$.0013 (the corresponding values for $Z^{\prime}$ is $\left.G^{\prime}= \pm 0 \$ 0011\right)$, and $\varepsilon= \pm 0 \$ 0072$. Standard deviations of (UT1 - UTC) $u$ (resolution interval is one day) and (UT1 - UTC) ${ }_{\text {BIH }}$ (resolution interval of five days) are $\sigma_{u}= \pm 0 \$ 0027$ (Djurovic, 1975b), and $\sigma_{\mathrm{BIH}}= \pm 0.0018$ (Guinot, 1976). From the comparison of $\sigma$ and $\varepsilon$ with $\sigma_{u}$ and $\sigma_{B I H}$ it appears that (UT1 - UTC) ${ }_{\text {PZT }}$ is equivalent to the mean systems of (UT1 - UTC) $u$ and (UT1 - UTC) ${ }_{\text {BIH }}$ with respect to accidental errors.

\section{CONCLUSION}

With the present number of PZTs the accuracy of the BIH five-day mean of polar coordinates is greater than that obtained from a solution using PZTs only. The present accuracy can be attained with a PZT solution if a longer averaging period were used (approximately ten days).

As individual PZT systems of (UTO - UTC) ${ }_{i j}$ are more homogeneous than (UT0 - UTC) $i j$ obtained from the observations of transit instruments and astrolabes (Djurovic, 1978), the mean system (UT1 - UTC) PZT $_{\text {is equiv- }}$ alent to the (UT1 - UTC) $\mathrm{BIH}$. The continuity of the classical observations of the Earth's rotation can be preserved by existing PZTs only. 


\section{REFERENCES}

Djurovic, D.: 1975a, Bul1. Obs. Astron. Belgrade 126, p. 62.

Djurovic, D.: 1975b, Bul1. d'Observations, Obs. Royal Belgium 4, fasc. 3, p. 17 .

Djurovic, D.: 1976, Bul1. Accad. Serbe Sc. et Arts 55, no. 9, p. 7.

Djurovic, D.: 1978, Pub1. Depart. Astron. Belgrade 8, p. 5.

Fliege1, H. F., and Hawkins, T. P.: 1967, Astron. J. 72, p. 544.

Guinot, B.: 1976, Report presented to IAU General Assembly, Grenoble.

Markowitz, W.: 1960, in S. K. Runcorn (ed.), "Methods and Techniques in

Geophysics", Intersciences Publishers Ltd., London.

Mihailov, A. A.: 1971, Astron. Zhurn. Akad. Naouk SSSR 48, no. 6, p. 1301.

Munk, W. H., and MacDonald, G. J. F.: 1960, "The Rotation of the

Earth", Cambridge University Press, London.

Pavlov, N. N., and Staritzin, G. V.: 1962, Astron. Zhurn. Akad. Naouk SSSR 39, no. 1, p. 123.

Popov, N. A.: 1963, Astron Zhurn. Akad. Naouk SSSR 7, p. 422.

Rochester, M. G., Jensen, O. G., and Smylie, D. E.: 1974, Geophys. J. Roy. Astron. Soc. 38, p. 349.

Stoyko, N.: 1938, Acta Astron. 3, p. 97.

Toomre, A.: 1974, Geophys. J. Roy. Astron. Soc. 38, p. 335. 